ARTICLE OPEN

\title{
Hall voltage drives pulsing counter-currents of the sliding charge density wave and of quantized normal carriers at self- filled Landau levels
}

\author{
Andrey P. Orlov ${ }^{1}$, Aleksander A. Sinchenko ${ }^{1}$, Pierre Monceau ${ }^{2,3}$, Serguei Brazovskii ${ }^{4,5}$ and Yuri I. Latyshev ${ }^{1}$
}

Remnant pockets of carriers left over after formation of a charge density wave (CDW) were brought, by virtue of transverse electric and magnetic fields, to a current-carrying state at quantized Landau Levels. The generated Hall voltage polarizes and puts to sliding the flexible CDW background. The screening from the CDW allows for a so strong redistribution of normal electrons density under the action of the Lorentz force alone, that an integer filling of the lowest Landau level might be reached at one edge at the expense of the full depletion at another edge of the Hall bar. With the Hall field exceeding the sliding threshold, the regime of exactly compensated collective and normal counter-currents develops in the open-circuit direction across the bar. The annihilation of the two currents proceeds via a regular sequence of phase slips which are the space-time vortices of the CDW phase around the enforced amplitude nodes. The resulting spontaneous generation of coherent high $(\sim \mathrm{GHz})$ frequency signals was detected by observations of multiple Shapiro steps. This picture results from studies of micron-sized Hall bars in crystals of $\mathrm{NbSe}_{3}$ prepared by means of focused ion beams. The interpretation is confirmed and illustrated by a numerical solution of the derived equations. The depinning pulse propagates from edges to the bulk and the sliding sets in, accompanied by the generation of periodic phase slips near the Hall bar edge where the CDW phase is advanced in steps of $2 \pi$ at expense of the CDW amplitude passing through zero.

npj Quantum Materials (2017)2:61 ; doi:10.1038/s41535-017-0070-3

\section{INTRODUCTION}

Charge density waves (CDW) are broken-symmetry states ubiquitous in low-dimensional electronic systems. CDWs are directly observed as concomitant periodic modulations of the electronic density and of atomic distortions $\propto \cos (\vec{Q} \vec{r}+\varphi)$ characterized by the wave vector $\vec{Q}$. The CDW is a macroscopic quantum state ${ }^{1}$ described by a complex order parameter $\Psi \sim A e^{i \varphi}$ where $A$ and $\varphi$ are respectively the amplitude and the phase of the CDW. The approximate degeneracy with respect to displacements of $\varphi$ gives rise to the most remarkable effects of the CDW sliding. The time dependence of $\varphi$ determines the collective electric current $j_{c} \propto \partial \varphi / \partial t$ which can be accompanied by the spontaneous generation of a coherent periodic signal with the same frequency $f=\partial \varphi / \partial t /(2 \pi)$.

In conventional experiments on CDWs (see reviewers ${ }^{2,3}$ ), the driving force for the depinning and the sliding is provided by the electric field, $E_{x}$ in the direction $x$ of CDW chains. The penetration of $E_{x}$ originated by the applied voltage $V_{x}$ is mediated by the normal current density, $j_{n x}$; so the sliding and the normal currents flow in the same direction $x$. The initial motivation of the present studies was to show that the CDW can also be driven by the Hall voltage $V_{H}$ originated from the normal current passing in a transverse direction. That has been proved indeed as we shall see below, but the observed effects went well above the best anticipations.
Here we shall show that, thanks to the highly polarizable CDW background, the remnant normal carriers under the quantized magnetic field and the imposed transverse electric current can be put first into the regime of a self-tuned nearly integer quantum Hall (IQH) state. With increasing current, that is followed by the sliding regime of the pulsing collective current which is exactly compensated by the counter-current of normal electrons. The zero-sum loop of these two currents is closed by periodic phaseslip processes ${ }^{4}$ giving rise to spontaneous coherent oscillations observed up to the $\mathrm{GHz}$ range.

This complex phenomenon essentially integrates the coherency of the collective ground state and the extreme quantum regime of the "normal" component. In $\mathrm{NbSe}_{3}$ the normal current is carried by remnant carriers in small pockets of electron and hole types. Their characterization is still incomplete and even contradictory in some respects, particularly intriguing in high magnetic fields (see latest results and discussion in ref. 5 and the Supplementary Discussion 1). Still, there is enough information certifying that in a magnetic field $B \approx 20 T$ the electrons and holes are fully quantized occupying only fractions $\nu \sim 10^{-1}$ of their lowest Landau levels.

\section{RESULTS}

Experimental

With the help of the focused ion beam (FIB) technique we have designed a Hall bar scheme by cutting out a narrow channel

\footnotetext{
${ }^{1}$ Kotel'nikov Institute of Radioengineering and Electronics of RAS, Mokhovaya 11-7, 125009 Moscow, Russia; ${ }^{2}$ Université Grenoble Alpes, CNRS, Grenoble INP, Institut Néel, 38000

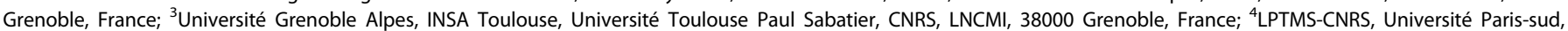
Université Paris-Saclay, 91405 Orsay, France and ${ }^{5}$ National University of Science and Technology "MISiS", Moscow 119049, Russia

Correspondence: Aleksander A. Sinchenko (aasinch@mail.ru) or Pierre Monceau (pierre.monceau@neel.cnrs.fr)
}

Received: 27 February 2017 Revised: 3 October 2017 Accepted: 18 October 2017

Published online: 06 November 2017 


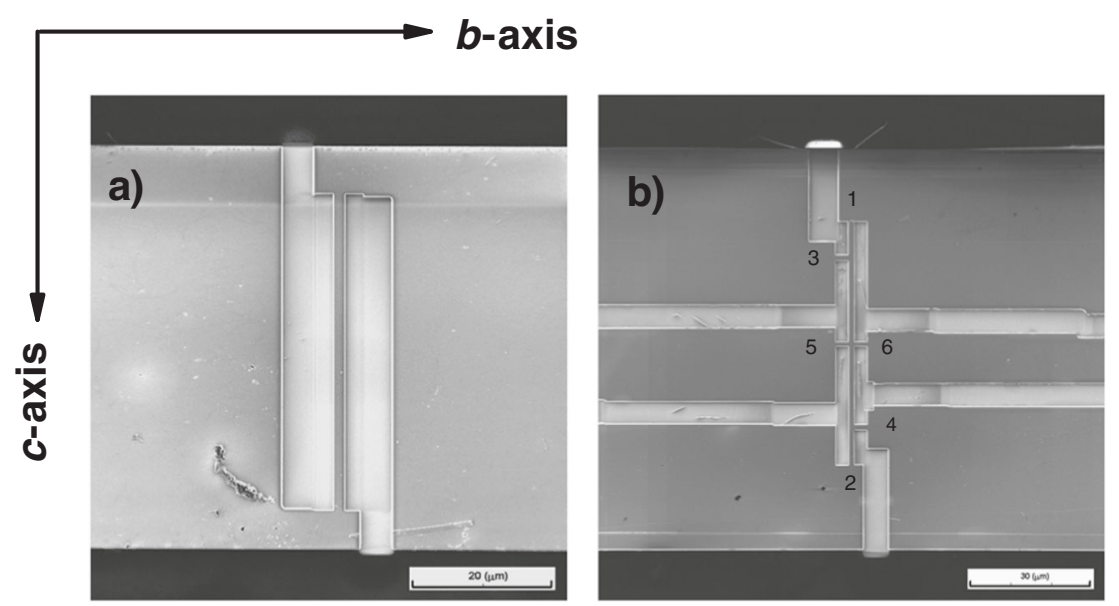

Fig. 1 Bridge-type structures along the $c$-axis (across the chains) prepared on thin $\mathrm{NbSe}_{3}$ single crystals using FIB: a two probes structure; b six probes structure with different distances between potential probes (contacts $3-4 ; 3-5 ; 6-4$ ) and containing the Hall probes (contacts $5-6$ ). The width of the channels varies from 1 to $6 \mu \mathrm{m}$ and the length from 20 to $43 \mu \mathrm{m}$

transversely to the chains' "b" ( $x$ axes) direction of the $\mathrm{NbSe}_{3}$ whisker crystal as shown in Fig. 1. The bias current was passed along the channel in the " $c$ " ( $y$ axes) direction, the magnetic field $B$ was applied in another transverse direction "a" ( $z$ axes), perpendicular to the highly conducting $(b, c)$-plane, for the purpose to generate the Hall voltage $V_{H}$ along the chain axis.

Figure 1 shows two different fabricated bridge-type structures. The channels, for which length $L$ and width $W$ varied from 20 to $43 \mu \mathrm{m}$ and from 1 to $6 \mu \mathrm{m}$ correspondingly, were oriented across the conducting chains, along the crystal $c$-axis. Totally 8 structures have been prepared and studied. We used two geometrical configurations: the two-probes structure shown in Fig. 1a (sample Nos. 1, 3-8) and the six-probes structure (sample No.2) with different distances between potential probes and containing the Hall probes (Fig. 1b).

Temperature dependence of the channel resistance in these structures (see Fig. S1a in Supplementary Methods 2) resembles the $R_{y y}(T)$ one obtained from bulk measurements. ${ }^{6}$ In Fig. S1b we show the field dependencies of the resistance $R_{y y}(B)$ and the Hall voltage, $V_{x}(B)$, at $T=4.2 \mathrm{~K}$ for the same sample. The plot of $R_{y y}(B)$ demonstrates the gigantic magnetoresistance which increases more than 20 times by $B=20 T$. The Hall field $V_{x}(B) / W$ is a linear function of $B$ (except for low fields $B<0.57$ ). For the bias current $I$ $=10 \mu \mathrm{A}$ it reaches $\sim 1.5 \mathrm{~V} / \mathrm{cm}$ which is comparable with the bulk value of the threshold electric field $E_{x t}$ for the onset of the CDW sliding at this temperature.

Figure $2 a$ shows a set of differential $I-V$ curves for sample No. 4 under various magnetic fields $B$. They are nearly flat at $B<6 T$, but at higher fields a threshold feature appears in $d I / d V(I)$ and sharpens with the field increase. Above the threshold, the differential conductivity sharply drops down (by 3-5 times at $B$ $=207$ ). Each curve was swept from zero to the maximum current $+I_{\text {max }}$ then backwards to $-I_{\max }$ and finally to $I=0$ with no hysteresis observed.

Figure $2 b$ shows that the threshold feature for sample No.1 is smeared out with temperature and it nearly disappears above 30 K. The threshold current, however, is nearly temperature independent, at least for $B=20 T$. From Fig. $2 \mathrm{a}$, $\mathrm{b}$ it follows that the nonlinearity in $I V$ characteristics appears only at high magnetic field and at low temperatures.

Figure 2c shows $d I / d V(I)$ dependencies at $B=20 T$ for two channels of different lengths, 20 and $30 \mu$ (samples 4 and 5). This pair of structures was fabricated from the same single crystal, so that the thickness $D$ was kept constant. It follows that the threshold current is independent of the channel length but the magnitude of the conductivity drop is directly proportional to the length.

The value of the Hall voltage was directly measured with the Hall probes of the structure shown in Fig. 1b. We measured the dependence of the differential Hall resistance, $R_{x y}=d V_{x} / d l$, as a function of the applied current simultaneously with the differential $I V$ characteristic $R_{y y}=d V / d l$ along the $c$-axis. As shown in Fig. $2 \mathrm{~d}$, the $R_{x y}$ does not change through the critical current where $1 / R_{y y}$ shows a sharp drop.

The nature of the sharp anomaly in the nonlinear transport across the chains becomes elucidated when we find that the state at $I>I_{\mathrm{t}}$ is accompanied by the emission of a coherent electromagnetic radiation. That was proved by observing on the $I-V$ characteristics the Shapiro-steps type response to an external radiation in the $\mathrm{MHz}-\mathrm{GHz}$ frequency range. Fig. 3a shows that under the $\mathrm{RF}$ radiation the plateau at $|I|<I_{\mathrm{t}}$ sharpens into the zero bias peak which is not surprising since the RF amplitude well exceeds the dc threshold. Most striking is the appearance of a sequence of multiple peaks in $d I / d V(V)$ which are nearly equidistant in voltage, like in superconductors, rather than in current as in CDWs. The peaks might correspond to the matching of the external frequency $f_{\mathrm{ex}}=f$ and the intrinsically generated frequency $f_{\text {int }}$ in the system when $f_{\text {ex }}=(p / q) f_{\text {int }}$, with integers $p$ and $q$. The condition $p / q=1$ determines the fundamental Shapiro step $\Delta V$.

The position of the fundamental Shapiro step is shown to be proportional to the external frequency (Fig. 3a) and also to the length of the channel, but it does not depend either on the thickness or on the width of the bridges. The generic linear dependence $\Delta V \propto f$ on the frequency was observed for 7 structures of different lengths. Note also that the Shapiro step structure is absent in the differential Hall conductivity.

In Fig. $3 \mathrm{~b}$ the period of voltage peaks is shown for the value $\Delta V /$ $N_{\mathrm{c}}$ normalized to the number of chains $N_{\mathrm{c}}$ which reduces the total voltage to the interchain voltage drop. From these data we can calculate $\Delta V / f=12.5 \mathrm{mV} / \mathrm{GHz}$ for $1 \mu \mathrm{m}$ of the structure length. According to the crystalline structure of $\mathrm{NbSe}_{3}, 1 \mu \mathrm{m}$ along the $c$ axis corresponds to 641 cells or $N_{c}(1 \mu \mathrm{m})=641 \times 2$ chains if we consider that the low-T CDW appears on a group of two chains within the cell. Then we get $\Delta V /\left(N_{c} f\right)=10 \mu \mathrm{V} / \mathrm{GHz}$ per chain which is 4 times the Josephson relation $\Delta V / f=2.5 \mu \mathrm{V} / \mathrm{GHz}$ in superconductors.

Evolution of the Shapiro steps structure with temperature is shown in Fig. 3c. As the temperature increases, the amplitude of steps decreases and $\Delta V$ increases (Fig. 3d). The Shapiro steps structure completely disappears above $T \approx 30 \mathrm{~K}$. More details on 

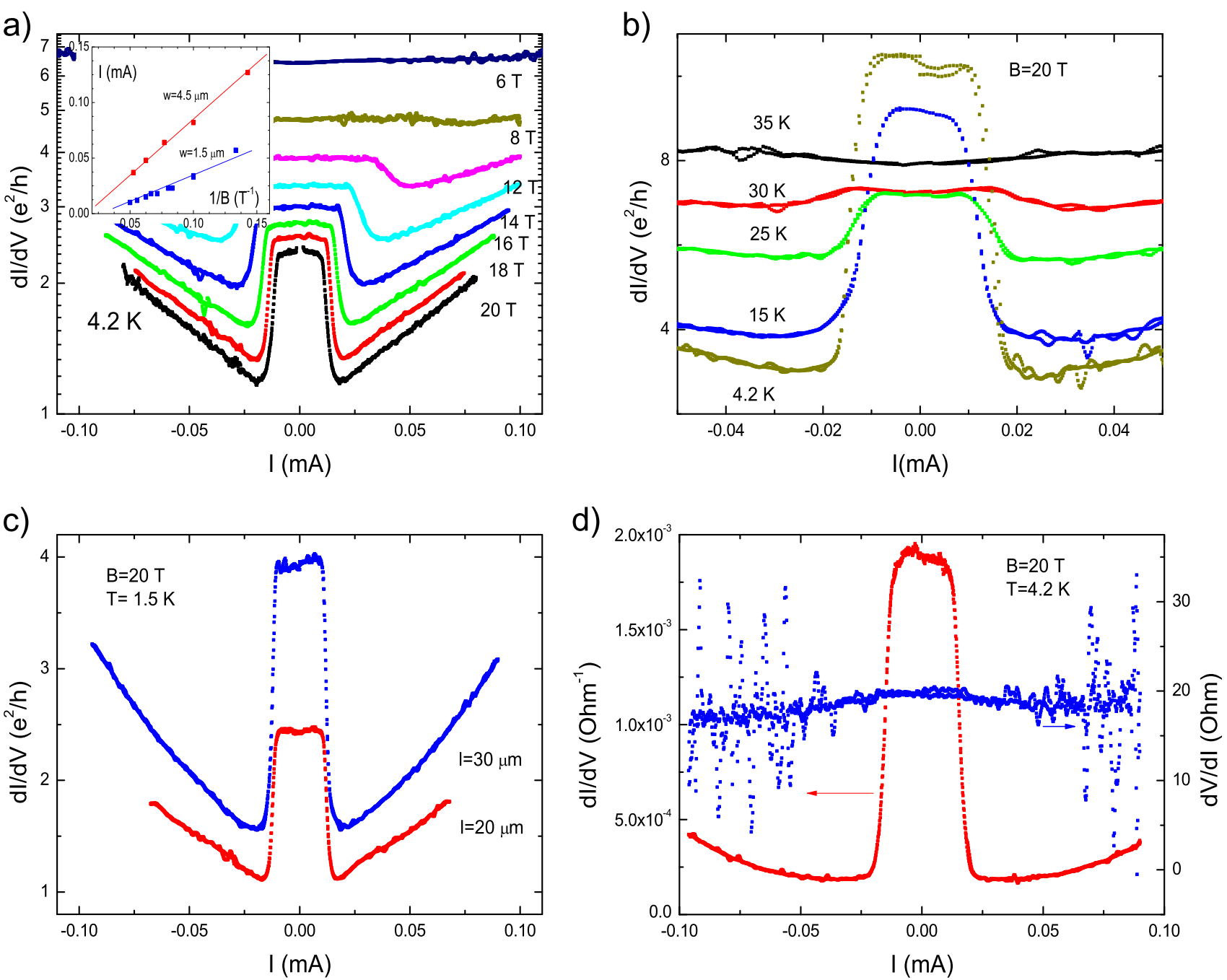

Fig. $2 I-V$ characteristics at various temperatures and magnetic fields. a $d I / d V$ dependencies on $/$ in $e^{2} / h$ units along the $c$-axis: a At various $B$ for $T=4.2 \mathrm{~K}$ (sample No. 4). The inset shows the variation of the threshold current vs. $1 / B$ for channels with widths 1.5 and $4.5 \mu \mathrm{m}$ (samples 2 and 8). b For several temperatures at the fixed $B=20 \mathrm{~T}$ (sample 1). c At $B=20 \mathrm{~T}$ and $T=1.5 \mathrm{~K}$ for channels of different lengths, 20 and 30 microns, and the same width (samples 4 and 5). d $1 / R_{y y}$ dependence (red curve) and $d V_{x} / d I(I)=R_{x y}$ along the $b$-axis (blue curve) in the same range of current variation at $T=4.2 \mathrm{~K}$ and $B=20 \mathrm{~T}$ for sample No. 2

measuring of Shapiro steps can be found in the Supplementary Discussion 2.

The observed properties are completely different from the standard ones when the common CDW sliding is driven by the electric field in the chains direction. ${ }^{2,3}$ Here, the conductivity across the chains drops down above the threshold current, the Shapiro steps are quantized in voltage rather than in current, and these properties appear only in high magnetic field and at low temperature. The threshold current is independent on the length of the channel and varies as $\propto 1 / B$.

\section{Theoretical picture}

At the basic level of interpretation, the presented data are convincing that we are dealing with a depinning and subsequent sliding of the CDW under the generated Hall voltage. Indeed, the mutually orthogonal magnetic field and the bias current, both lying in transverse interchain directions, should produce the Hall voltage, $V_{x}$ in the chains direction. The Hall electric field is $E_{\mathrm{H}}=V_{x} /$ $W=R_{\mathrm{H}} I B /(D W)$ where $R_{\mathrm{H}}$ is the Hall constant, $D$ is the sample thickness. The threshold current $I_{\mathrm{t}}$ corresponding to the critical Hall electric field, $E_{\mathrm{Ht}}$ is $I_{\mathrm{t}}=E_{\mathrm{H} t} W D /\left(R_{\mathrm{H}} B\right) \propto W / B$ which reproduces the experimental dependencies on $W$ and $B$ (recall the inset in
Fig. 2a). The value of $V_{x}$ was directly measured using Hall probes of the structure shown in Fig. $1 \mathrm{~b}$ which yields the values like $E_{\mathrm{Ht}}=0.3$ $\mathrm{V} / \mathrm{cm}$ at $B=20 T$ and $T=4.2 \mathrm{~K}$ which is on the right scale of threshold fields in the bulk. Still, this interpretation invoking the CDW sliding faces a vital challenge: the circuit is open in the $x$ direction, hence the total current across the Hall bar must be zero. The collective CDW current must be compensated by a countercurrent of normal carriers, the picture of which is elaborated theoretically below, with more details given in Supplementary Notes 2-4.

Under the HMF but at no applied current yet, the carriers are distributed homogeneously forming a fractionally $(v<1)$ filled quantized state. (The value of $v<1$ is estimated as $v \approx 0.5-0.1$ at $B$ $=20 T$, see Supplementary Discussion 1.) When the transverse current $J_{y}$ is applied, the Lorentz force in $x$ direction appears pushing the carriers orbits towards one end (which we choose as $x=0$ ) until the charge redistribution gives rise to the compensating Hall field $E_{x}$. The first key peculiarity with respect to conventional Hall setup is the presence of the CDW background with its gigantic polarizability, estimated here as $\epsilon \propto 10^{6}$ (see Supplementary Note 1). The screening by displacements of the CDW phase $\varphi \propto \epsilon E_{x}$ allows for a strong redistribution of the 
a)

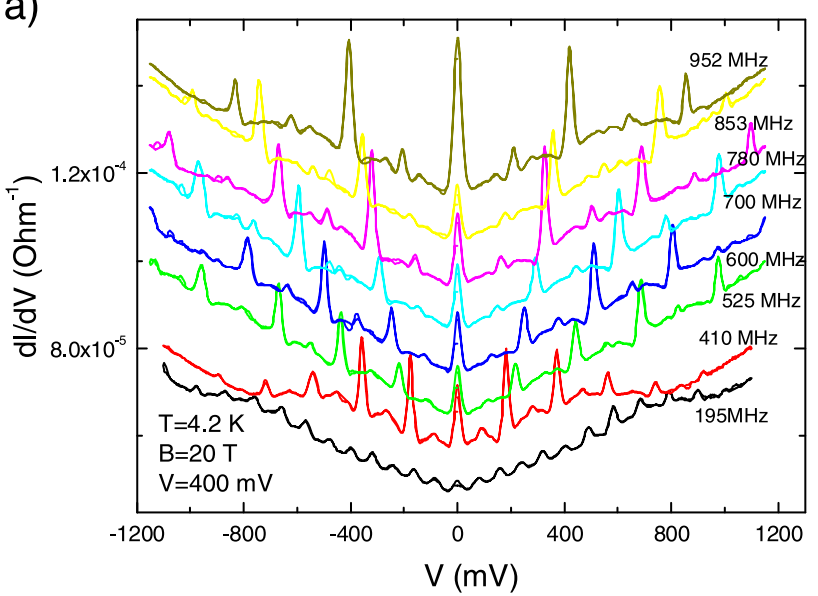

b)

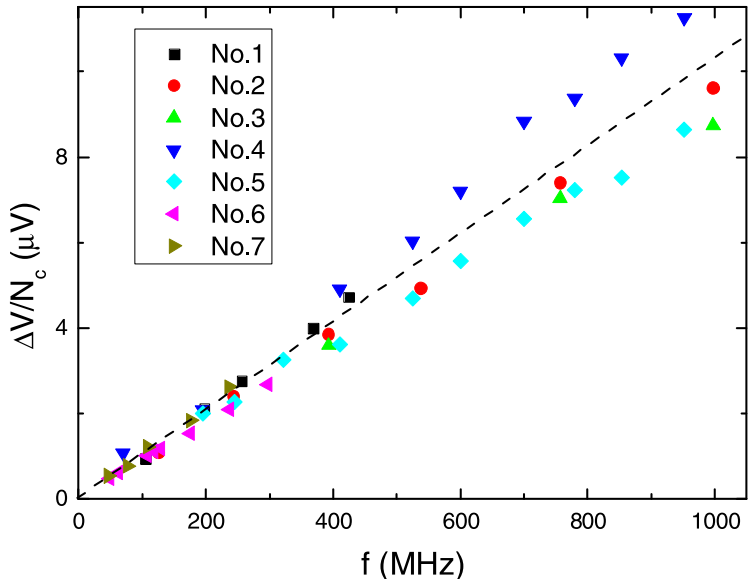

c)

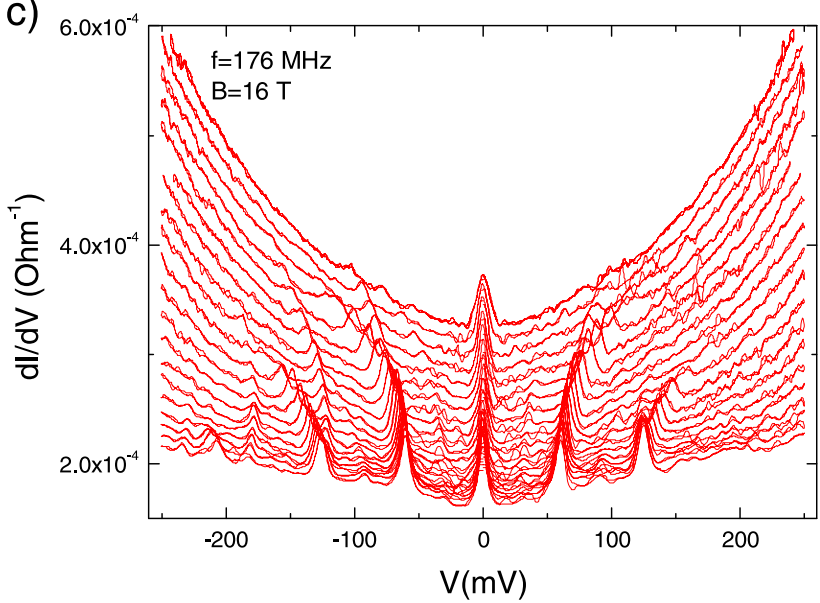

d)

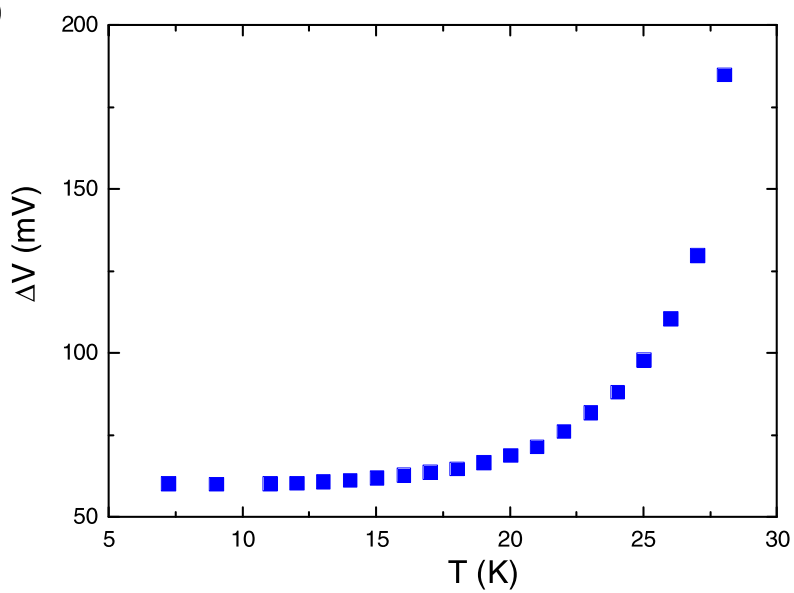

Fig. 3 Shapiro steps. a Shapiro steps response of the structure No. 4 to the application of an external RF-field for various frequencies of the same amplitude $400 \mathrm{mV}$ at $B=20 T$ and $T=4.2 \mathrm{~K}$. b Variation of the interchain voltage $\Delta V$ vs. frequency of an external radiation $f$ for all measured structures. c $d I / d V(I)$ for different temperatures at $B=16 T$ and the external frequency $f=176 \mathrm{MHz}$ (sample No. 7). Temperature increases from $7 \mathrm{~K}$ (lower curve) up to $29 \mathrm{~K}$ (upper curve) with the step $\Delta T=1 \mathrm{~K}$. d Temperature variation of $\Delta V$

a)

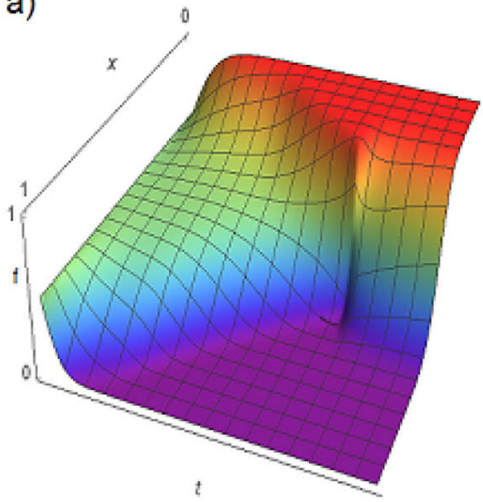

b)

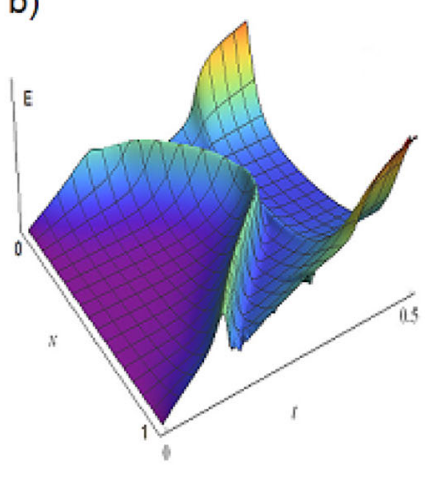

c)

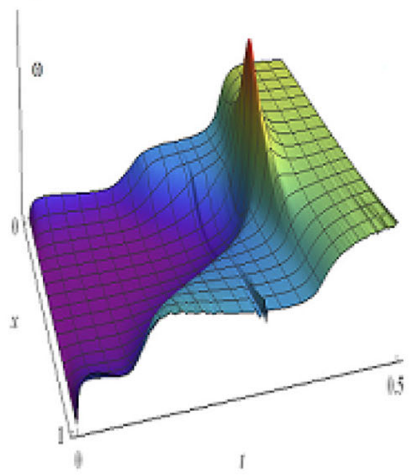

Fig. 4 Space-time evolution of the depinning event: occupation numbers $f_{\mathrm{F}}(x, t)(\mathbf{a})$, the electric field $E=E_{x}(\mathbf{b})$, and the phase velocity $\partial_{t} \varphi(\mathbf{c})$

electronic density, see Fig. 4, up to (in the $T=0$ limit) the complete charge segregation when all carriers occupy, with the maximum filling 1 , a segment $I=v L_{x}$ of the chain length $L_{x}$, thus forming here the $\mathrm{IQH}$ state while leaving the segment $(1-v) L_{x}$ unoccupied.

The electric field accompanying such a charge separation easily exceeds the pinning threshold for the CDW sliding. Then, as we demonstrate in Fig. 4 and in Supplementary Note 3, Sec. 4, the depinning propagates into the nominally pinned central region via sharp walls. The resulting picture is that of compensated collective $j_{c}$ and normal $j_{n x}$ pulsing counter-currents driven by the Hall voltage. The appearance of $j_{n x}$ gives rise to the secondary Hall voltage, now in the $y$ direction, the persistent part of which is 
registered as a sharp threshold in the $I-V$ curve while the periodic part gives rise to Shapiro steps. This scenario will be illustrated below by a numerical modeling.

In the regime of the extreme Landau quantization, the kinetic energy is no more involved, then the density is determined locally by the electric potential $\Phi(\vec{r})$. The profile for the density of carriers per chain is $n_{1}(x)=f_{\mathrm{F}}(\Phi(x) / T) \bar{n}_{1} / \nu$ where $\nu=(h c / e B)\left(\bar{n}_{1} / d_{y}\right)$, $\bar{n}_{1}=N_{1} / L_{x}$ is the mean concentration of carriers per unit length of one chain, $d_{y}$ is the interchain distance, and $f_{\mathrm{F}}(z)$ is the Fermi distribution function. Integration over $x$ of the one-particle transverse current density $e c E_{x}(x) / B$, weighted with $n_{1}(x)$, yields the overall current as a function of values of the potentials only at $x$ boundaries:

$\frac{J_{y}}{J_{T} N_{\mathrm{a}}}=\ln \frac{1-f_{\mathrm{F}}\left(\Phi\left(L_{x}\right) / T\right)}{1-f_{\mathrm{F}}(\Phi(0) / T)}, J_{T}=\frac{e}{h} T \approx 3 \times 10^{-3} \mu \mathrm{A} \times T[K]$

where $N_{\mathrm{a}}$ is the number of $(b, c)$ planes across the sample height. Above the threshold, the CDW starts sliding, carrying on the collective current $J_{c}$, which must bring about the compensating normal counter-current $J_{n x}=-J_{c}$ since the total $x$ current must be zero. Then the new component of the Lorentz force appears in the $y$ direction giving rise to the reciprocal Hall voltage $V_{y}$. Its magnitude is given by the universal law similar to Eq. (1) with interchanged indices $x$ and $y$ (and the opposite sign). For the current per chain we get

$j_{x 1}=-\frac{e}{h} \frac{d_{y}}{L_{y}} \int_{0}^{L_{y}} \partial_{y} \Phi(x, y) f_{\mathrm{F}}(\Phi(x, y)) d y \approx \frac{e^{2}}{h} \Delta V_{y}(x)$

where $\Delta V_{y}(x)=\left(\Phi(x, 0)-\Phi\left(x, L_{y}\right)\right) d_{y} / L_{y}$ is the mean interchain voltage drop. The charge separation was important for this conclusion: in Eq. (2) the integration by $y$ was carried on over the line close to the edge $x=0$ where $f_{\mathrm{F}}(\Phi(x, y)) \approx 1$.

The last step is to remind that the CDW countercurrent, per chain, is $j_{c 1}=-e \partial_{t} \varphi / \pi$ and it requires phase slips ${ }^{4}$ to annihilate with the normal current $j_{x 1}$ near the boundary. Let each phase slip absorb/release $M$ electrons per chain, then the repetition frequency is

$f=\frac{|\dot{\varphi}|}{2 \pi M}=\frac{\left|j_{x 1}\right|}{2 M e} \approx \frac{e\left|\Delta V_{y}\right|}{2 M h}, \frac{\left|\Delta V_{y}\right|}{f}=\frac{2 M h}{e}$

We arrive at the Josephson type relation with an additional factor $M$; according to experiments, with a good accuracy being integer $M \approx 4$. The phase slip appears to be a spacio-temporal supervortex, with the $M$-fold circulation. This value may be related to the number of normal carriers within the chain segment which are available for transformation to/from the condensate within each phase slip.

The sharp strong drop of the transverse normal current $J_{y}$ with the onset of the loop currents in the chains' direction can also be illustrated by means of quite general phenomenological considerations which we present in Supplementary Note 2.

\section{The modeling}

The numerical work was performed on the bases of the timedependent Ginzburg-Landau approach the adaptation of which leads to nonlinear partial differential equations described in Supplementary Note 4. Here we shall present the most important results.

1. At the critical current, the time evolution starts with a depinning pulse propagating from edges to the bulk, after which the regime of the homogeneous sliding sets up. Results of the modeling (described in Supplementary Note 3) are shown in Fig. 4 for the lowest experimental temperature $1 \mathrm{~K}$. The plots demonstrate two propagating fronts of depinning originated (at different moments) at the boundaries where the electric field is enhanced (plot $4 \mathrm{~b}$ ) because of the charge redistribution (plot 4a). The fronts collide showing a strong brief peak of the phase velocity $\partial_{t} \varphi / \pi$ (plot 4c), and then annihilate giving rise to the overall homogeneously sliding state (plot 4c) while on the background of the very inhomogeneous distributions of the electric field and the particles' density. Initially, the distribution of occupation numbers is homogeneous $f_{\mathrm{F}}(x, 0)=v=0.5$. After passing of the first depinning front, the two plateaus with $f \approx 1$ and $f \approx 0$ are established giving rise to a nearly $\mathrm{IQH}$ region at the expense of the nearly unpopulated one.The rising of occupation numbers $f_{\mathrm{F}}(t, x)$ towards the channel edge $x=0$, up to the IQH limit $f_{\mathrm{F}}(t, 0)=1$, can be understood already from the most general relation (1). Indeed, the value $J_{T} N_{a} \approx 3 \mu \mathrm{A}$ (with $N_{\mathrm{a}} \approx 1000$ ) corresponds to the lowest experimental currents while in most important regimes $J_{y} /\left(J_{T} N_{a}\right) \propto 10^{2}$ is big. Then (1) requires inevitably that $f_{\mathrm{F}}(t, 0)=1$. To keep the mean value of $\left\langle f_{\mathrm{F}}\right\rangle_{x}=\nu$ over $x$, the compensating depletion should take place towards $x=L_{x}$.

2. At higher currents the regime of a totally depinned sliding sets in, which is accompanied by the generation of periodic phase slips-the events where the CDW phase is advanced in steps of $2 \pi$ or $2 M \pi$ with integer $M$. They allow to detach the fast sliding high-field stripe near $x=0$ from the slowly sliding body.

Results of the modeling (described in Supplementary Note 4) are presented in Fig. 5. The phase slip is a vortex of the CDW complex order parameter $\psi$ in space-time coordinates $(x, t)$; hence the amplitude node $A(x, t)=0$ develops in the vortex center. The amplitude does not recover completely in-between the nodes, so its standing depression is formed from which $A(x, t)$ shoots instantaneously further down to zero. This periodic sequence might have given rise to the experimentally observed Shapiro steps.

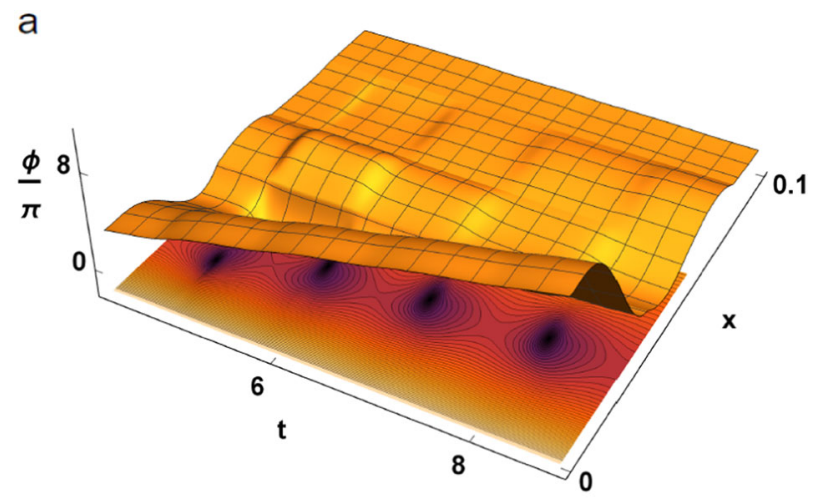

b 0.1

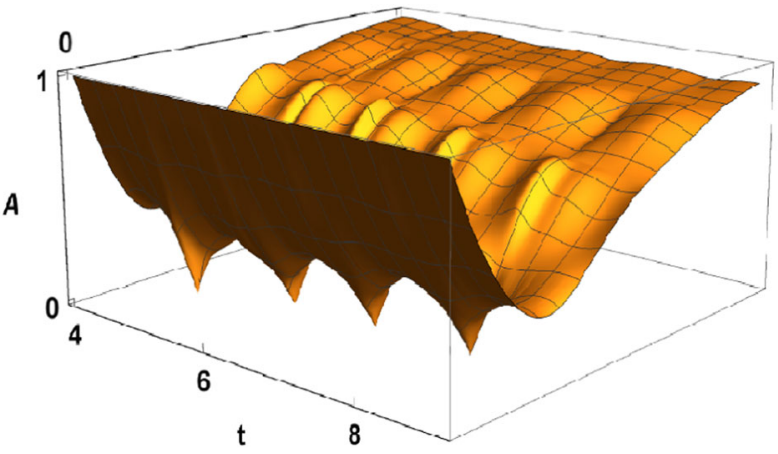

Fig. 5 Plots of spatiotemporal evolution with periodic phase slips: a the 3D plot of the phase $\varphi(t, x) / \pi)$ on top of the density plot of the amplitude $A(t, x) ; \mathbf{b}$ the CDW amplitude $A(t, x)$ (normalized to unity) 


\section{DISCUSSION}

Studies of density waves under high magnetic fields have already brought a firework of effects. Most known is the cascade of phase transitions among the Hall-quantized "field induced density waves" (see the reviews' collection ${ }^{8}$ ). Closer to the present work, the magnetic field could suppress the strong zero-bias conductance peak in $I-V$ characteristics of CDW mesa-structures which allowed to recover the physics of solitons and of electronic vortices $s^{9,10}$ in experiments on the internal tunneling.

Spontaneous superstructures of a $2 \mathrm{D}$ electron gas in a quantized magnetic field have already attracted much attention in experiment and theory, see a short review in Sec. 11.6.4. of ref. 3. They have been identified as Wigner crystals (see reviews ${ }^{11,12}$ ) and as a richness of bubble, stripe, and nematic states (see reviews ${ }^{13,14}$ ). These structures have been registered and explained as ground states of electronic systems in the magnetic field, with the collective sliding showing up as a posteriori effect. The state described in this article is fundamentally non static. It appears, under the effect of the Lorentz force, as a stationary state carrying the Hall current. At higher bias currents above the threshold it develops as an essentially dynamical pulsating state.

In conventional sliding CDWs, the normal current injected at junctions is gradually transformed into the collective one in the bulk, and this process is assisted by distributed phase slips. ${ }^{15,16}$ In the experiments reported here, the exactly compensated partial currents flow oppositely across the open-circuit Hall bar. By analogy, we recall observations ${ }^{17}$ of a CDW driven contactlessly by the thermopower which also assume the exact compensation of the normal and the sliding currents.

The intriguing part of the story are the phase slip processes understood as space-time vortices of the CDW complex order parameter. Their regular sequence is clearly observed via the Shapiro steps. Here the phase slips appear under unique circumstances of segregation of the electronic density among the plateaus of maximally filled and totally depleted quantized Landau levels. The modeling confirms that at the overcharged edge the integer quantum Hall state is self-tuned even if integrally, via measurements of the resistivity tensor, we do not observe the required integer characteristics of the quantum Hall state (because of the fractionally filled transient bulk region). Experiments at lower temperatures or higher magnetic fields should reach a truly stepwise boundary among the full and the zero field quantum levels. Such an improbable strong deviation of the electronic density from its mean value under the normally small Lorentz force became possible here because of the charge compensation by the gigantic polarization of the CDW, with the help of the FIB design allowing for high currents along the Hall bar.

In summary, the combination of the FIB fabrication of microscopic devices, application of a high magnetic field, and the modeling has allowed to identify the never seen regime of two-fluid quantum magneto-hydro-dynamics with compensated collective and normal conduction in the frame of the $\mathrm{QH}$ regime. The basic ingredient of this highly nonlinear and nonstationary regime is a sequence of periodic phase slip processes providing the annihilation of the normal and the collective currents. Beyond the generation of sliding pulses, the CDW plays the role of a screening medium which allows the Lorentz force to spaceseparate the mean non-integer density of normal electrons from the depleted zone to the one where the IQH state is self-tuned. The major role is played by ultralight carriers, ${ }^{5}$ suspected to be Weyl fermions, the emergence of which in $\mathrm{NbSe}_{3}$ from the ground state reconstruction by the CDW is a new tool in manipulating a quantum material.

\section{METHODS}

Measurements of $I V$ characteristics and their derivatives have been performed in conventional 4-probe configuration using computer controlled current source and nano-voltmeter. For studying nonstationary effects a RF current was superposed upon the dc current using the current contacts connected to the RF generator via two capacitors. Experiments in magnetic field up to $B=20 T$ have been carried out in a Bitter coil and in the superconducting magnet in National Laboratory of High Magnetic Fields in Grenoble. The structure was prepared by means of the focus ion beam (FIB) machine SMI-3050, Seiko Inc., Japan, with the resolution of Gaion beam of $6 \mathrm{~nm}$ in the regime of low current of few tens of pA. The electrical contacts to the crystal have been made by pressing indium stabs to the golden contact pads prepared by laser evaporation before the FIB process. More details on the material, methods and samples are described in SI: Supplementary Methods 1 and 2 for the experimental work and Supplementary Notes 3 and 4 for the theory and modeling.

Data availability

All relevant data are available from the authors.

\section{ACKNOWLEDGEMENTS}

Work at Grenoble was partially performed in the frame of the CNRS-RAS Associated International Laboratory between Institute Neél and IRE Physical properties of coherent electronic states in condensed matter. A.P.O. and A.A.S. acknowledge support from Russian State Fund for the Basic Research (No. 17-02-00665-a). S.B. acknowledges the financial support of the Ministry of Education and Science of the Russian Federation in the framework of Increase Competitiveness Program of NUST MISiS (N K3-2017033).

\section{AUTHOR CONTRIBUTIONS}

Y.I.L. (deceased) was at the origin of the mesa-junctions design and their studies in high magnetic fields. A.P.O. and A.A.S. carried out the experimental work and analysis; S.B. formulated the theoretical concept and performed the modeling; A.A.S. and P.M. coped with the experiment and, with S.B., wrote the paper. All authors contributed to discussions.

\section{ADDITIONAL INFORMATION}

Supplementary information accompanies the paper on the npj Quantum Materials website (https://doi.org/10.1038/s41535-017-0070-3).

Competing interests: The authors declare that they have no competing financial interests.

Publisher's note: Springer Nature remains neutral with regard to jurisdictional claims in published maps and institutional affiliations.

\section{REFERENCES}

1. Bardeen, J. Superconductivity and other macroscopic phenomena. Phys. Today. 43, 25-31 (1990).

2. Grüner, G. Density Waves in Solids (Addison-Wesley, Reading, Massachusetts, 1994).

3. Monceau, P. Electronic crystals: an experimental overview. Adv. Phys. 61, 325-581 (2012).

4. Gor'kov, L. P. Generation of oscillations by a running charge density wave. Sov. Phys. JETP 59, 1057-1064 (1984).

5. Sinchenko, A. A. et al. Hall effect in the pinned and sliding charge density wave state of $\mathrm{NbSe}_{3}$. J. Phys. Condens. Matter. 21, 435601 (2009).

6. Ong, N. P. \& Brill, J. W. Conductivity anisotropy and transverse magnetoresistance of $\mathrm{NbSe}_{3}$. Phys. Rev. B 18, 5265-5271 (1978).

7. Latyshev, Yu. I., Orlov, A. P. \& Monceau, P. Charge density wave transport in $\mathrm{NbSe}_{3}$ at low temperatures under high magnetic field. JETP Lett. 93, 99-102 (2011).

8. Physics of Organic Superconductors and Conductors, Vol. 110 Springer Series in Materials Sciences (ed. Lebed, A. G.) (Springer-Verlag, Berlin, Heidelberg, 2008).

9. Latyshev, Yu. I., Monceau, P., Brazovskii, S., Orlov, A. P. \& Fournier, T. Observation of charge density wave solitons in overlapping tunnel junctions. Phys. Rev. Lett. 95, 266402 (2005).

10. Latyshev, Y. I., Monceau, P., Brazovskii, S., Orlov, A. P. \& Fournier, T. Subgap collective tunneling and its staircase structure in charge density waves. Phys. Rev. Lett. 96, 116402 (2006). 
11. Fertig, H. A. Properties of the electron solid. In Perspectives in Quantum Hall Effects (eds Das Sarma, S. \& Pinczuk, A.) 71-108 (Wiley Interscience, New York, 1997).

12. Shayegan, M. Case for the magnetic-field-induced two-dimensional wigner crystal. In Perspectives in Quantum Hall Effects (eds Das Sarma, S. \& Pinczuk, A.) 343-384 (Wiley Interscience, New York, 1997).

13. Fogler, M. M. Stripe and bubble phases in quantum Hall systems. In High Magnetic Fields: Applications in Condensed Matter Physics and Spectroscopy (eds Berthier, C., Levy, L.-P. \& Martinez, G.) 98-138 arXiv:cond-mat/0111001 (SpringerVerlag, Berlin, 2002).

14. Fogler, M. M. Quantum Hall liquid crystals. Int. J. Mod. Phys. B 16, 2924-2929 (2002).

15. Lemay, S. G., van de Wijngaarden Lind, M. C., Adelman, T. L. \& Thorne, R. E. Spatial distribution of charge-density-wave phase slip in $\mathrm{NbSe}_{3}$. Phys. Rev. B 57, 12781-12791 (1998).

16. Requardt, $\mathrm{H}$. et al. Direct observation of CDW current conversion by spatiallyresolved synchrotron X-ray studies in $\mathrm{NbSe}_{3}$. Phys. Rev. Lett. 80, 5631-5634 (1998).

17. Beyermann, W. P., Mihaly, L. \& Grüner, G. Temperature-gradient-induced opencircuit electric currents in charge-density-wave condensates. Phys. Rev. Lett. 56, 1489-1493 (1986).
Open Access This article is licensed under a Creative Commons Attribution 4.0 International License, which permits use, sharing, adaptation, distribution and reproduction in any medium or format, as long as you give appropriate credit to the original author(s) and the source, provide a link to the Creative Commons license, and indicate if changes were made. The images or other third party material in this article are included in the article's Creative Commons license, unless indicated otherwise in a credit line to the material. If material is not included in the article's Creative Commons license and your intended use is not permitted by statutory regulation or exceeds the permitted use, you will need to obtain permission directly from the copyright holder. To view a copy of this license, visit http://creativecommons. org/licenses/by/4.0/.

(c) The Author(s) 2017 